\title{
Influences of Epidermal Cell Sizes and Flesh Firmness on Cracking Susceptibility in Sweet Cherry (Prunus avium L.) Cultivars and Selections
}

\author{
Masami Yamaguchi*, Isao Sato and Makoto Ishiguro \\ Yamagata Prefectural Horticultural Experiment Station, Sagae, Yamagata $991-0043$
}

\begin{abstract}
Summary
Susceptibility of cherry cultivars using water immersion method was investigated to determine the degree and rate of cracking, water absorption rate, fruit weight, flesh firmness, vertical and lateral lengths of the epidermal cells at the fruit apex, cheek, stalk cavity and suture. Thirty nine samples consisting of 37 sweet cherries, one sour cherry and one hybrid between sour cherry and Prunus pennsylvanica were used. The degree and rate of cracked fruit increased with time after immersion varied among cultivars: 'Turkey Black Heart', 'Jumbonishiki,' 'Okitama-6,' 'Ebony,' 'Venus' and 'Valera' were small, whereas 'Napoleon,' 'Van,' 'Compact Lambert' and 'Vic' were high. A huge variance occurred among cultivars with respect to fruit weight, flesh firmness, and vertical and lateral lengths of skin cells. After 12 hours of immersion, the following significant positive correlation coefficients were established between degree of cracking and fruit weight, flesh firmness, vertical and lateral lengths of skin cells at apex respectively; $0.664,0.515,0.649$ and 0.515 . Likewise, the rate of cracked fruit had significant positive correlations with fruit weight, flesh firmness, vertical and lateral lengths of skin cells at apex, respectively; 0.718, 0.503, 0.763 and 0.669 . Thus, cracking susceptibility is strongly related to fruit weight, flesh firmness and skin cell size, especially at the fruit apex. Significant positive relationships were also obtained between the water absorption rate, and flesh firmness and vertical length of the skin cells of the fruit apex. The contribution rates of cracked fruit rate and degree of cracking to all other parameters were 78.8 and $69.0 \%$, respectively. Likewise, high contribution rates of 70.7 and 57.3 $\%$ were obtained between fruit weight and flesh firmness to vertical length of the fruit apex. Hence, the possibility of estimating cracking susceptibility based on these three parameters is considered. Having obtained significantly high yearly correlation coefficients of $0.885,0.880$, 0.706 between degree of cracking, rate of cracked fruit and water absorption rate using 28 cultivars over a 2 -year period, the water immersion method to determine the degree of cracking was validated.
\end{abstract}

Key Words: cracking susceptibility, Prunus avium, skin cell size, water immersion.

\section{Introduction}

Fruit cracking and subsequent rotting is one of the most serious problems for sweet cherry (Prunus avium L.) growers in Japan because of rainy weather during harvest. To avoid these damages, cultivation under protected cover has increased; however, it is very expensive. From the perspective of the cherry industry worldwide, cracking of cherry is of great concern so that the development of crack-resistant cultivars is a high priority objective in breeding programs. Therefore, many researchers investigated the cracking mechanism (Verner and Blodgett, 1931; Tucker, 1934; Zielinski, 1964; Christensen, 1972a, b; King and Norton, 1987; Wade, 1988; Yamamoto et al., 1990a; Roser, 1996).

Received; January 7, 2002. Accepted; May 2, 2002.

* Corresponding author. Present address: National Institute of Fruit Tree Science. 2-1 Fujimoto, Tsukuba, Ibaraki 305-8605. E-mail: umetaro@affrc.go.jp
Cherry cracking is caused by the absorption of rainwater through the skin into the mesocarp, producing stress on the fruit surface and causing it to rupture (Verner and Blodgett, 1931; Tucker, 1934). For this reason, the influence of fruit surface structure or water pathway and stomata distribution to the water absorption level was studied (Sawada, 1931; Davenport et al., 1972; Iizuka and Yano, 1974). In addition, the relationship between changes in the cuticular layer versus fruit ripening and cultivation method was also investigated (Sekse, 1995). Factors accounting for variance in the cracking susceptibility of cherry cultivars were examined from different angles, c.g., soluble solids content and water storage capacity of fruit flesh (Kertesz and Nebel, 1935; Christensen, 1972b; Andersen and Richardson, 1982), calcium content (Lane et al., 2000) and other fruit flesh components, flesh cells, skin cells, size and structure of cuticular layer (Glenn and Poovaiah, 1989; Sekse, 1995; Lane et al., 2000), and regional stress distribution of fruit skin (Yamamoto et al., 1990b). However, the 
results of those investigations are conflicting because a the small number of cultivars was used in the studies, and the factors related to cracking are numerous. In the cherry fruit development, the growth of fruit is characterized by a rapid post-bloom cell division period of fruit flesh and skin, a relatively slow growth period, followed by a rapid fruit growth due to cell enlargement to harvest. In this process, stress on the skin, which accompanies fruit growth, may increase. We used as many cultivars as possible to examine the relationship between fruit weight and firmness (traits considered important in breeding), and cracking susceptibility to establish the relationship between cracking and skin cell size.

\section{Materials and Methods}

In 1990, 26 sweet cherry cultivars, one sour cherry and one hybrid (' $P$. cerasus $\times$ Pin') of sour cherry and a $P$. pennsylvanica selection from North America, grow-
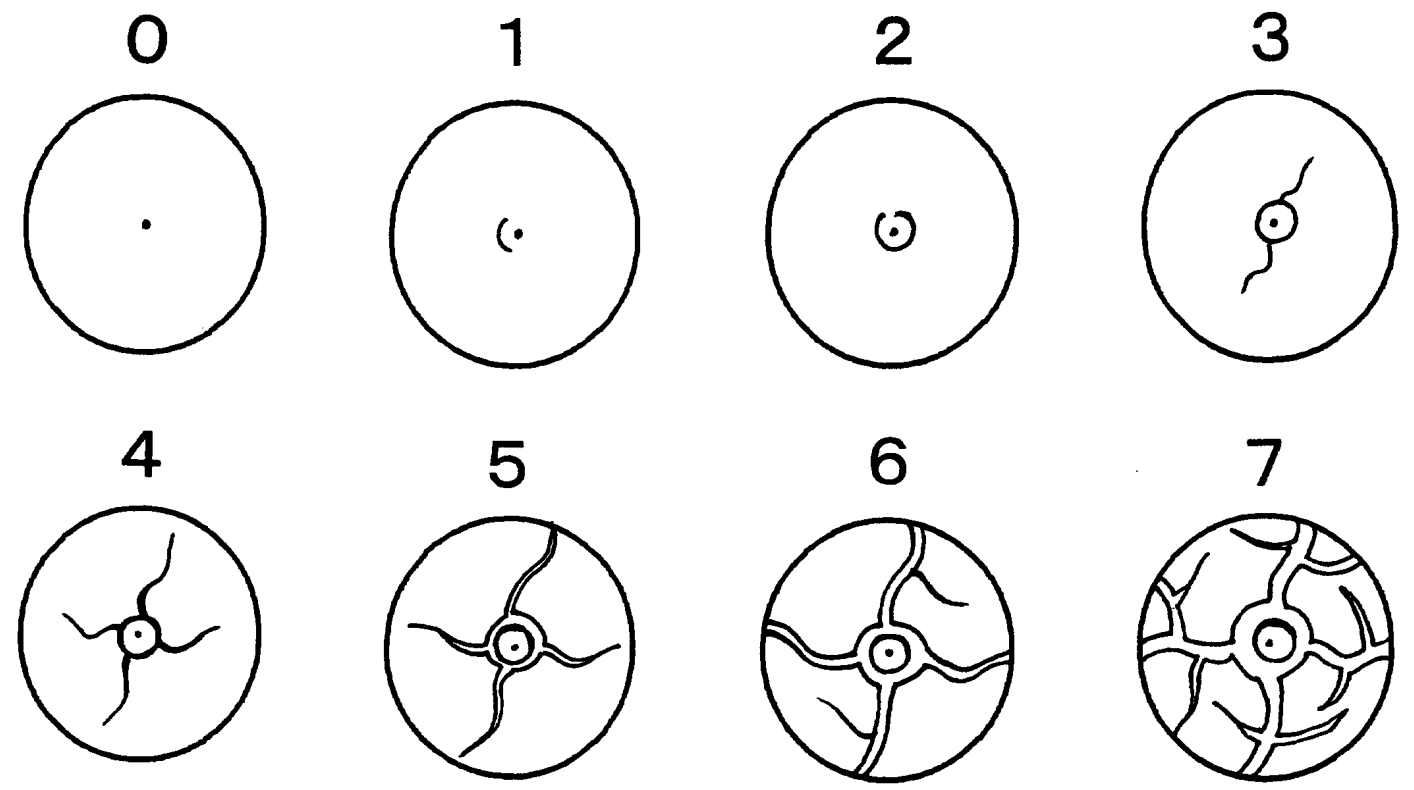

ing at Yamagata Prefectural Horticultural Exp. Sta. in Sagae, were used to study the changes in water absorption rate, degree of cracking and rate of cracked fruit through water immersion method. In the water immersion experiment, 20 mature fruit from each cultivar were divided into two groups, and each group was weighed and placed in a polyethylene bag, into which de-ionized water was added while allowing part of the fruit remains above the surface of the water to facilitate respiration. Each bag was placed in incubator kept at $20^{\circ} \mathrm{C}$; each fruit was weighed after 12, 24 and $48 \mathrm{hr}$. Degree of cracking was calculated as the average degree of cracking of the 20 fruit samples (Fig.1). The percentage of cracked fruit was recorded for each cultivar.

Based on the the data obtained from the immersion method in 1990, in 1991, 37 sweet cherry cultivars, one sour cherry, a hybrid between a sour cherry and $P$. pennsylvanica were examined. Furthermore, the relationship between cracking susceptibility and fruit skin

Fig. 1. Patterns and degree of cracking of cherry fruit classified after a scale rating from $0=$ no visible cracking to $7=$ extremely severe cracking.
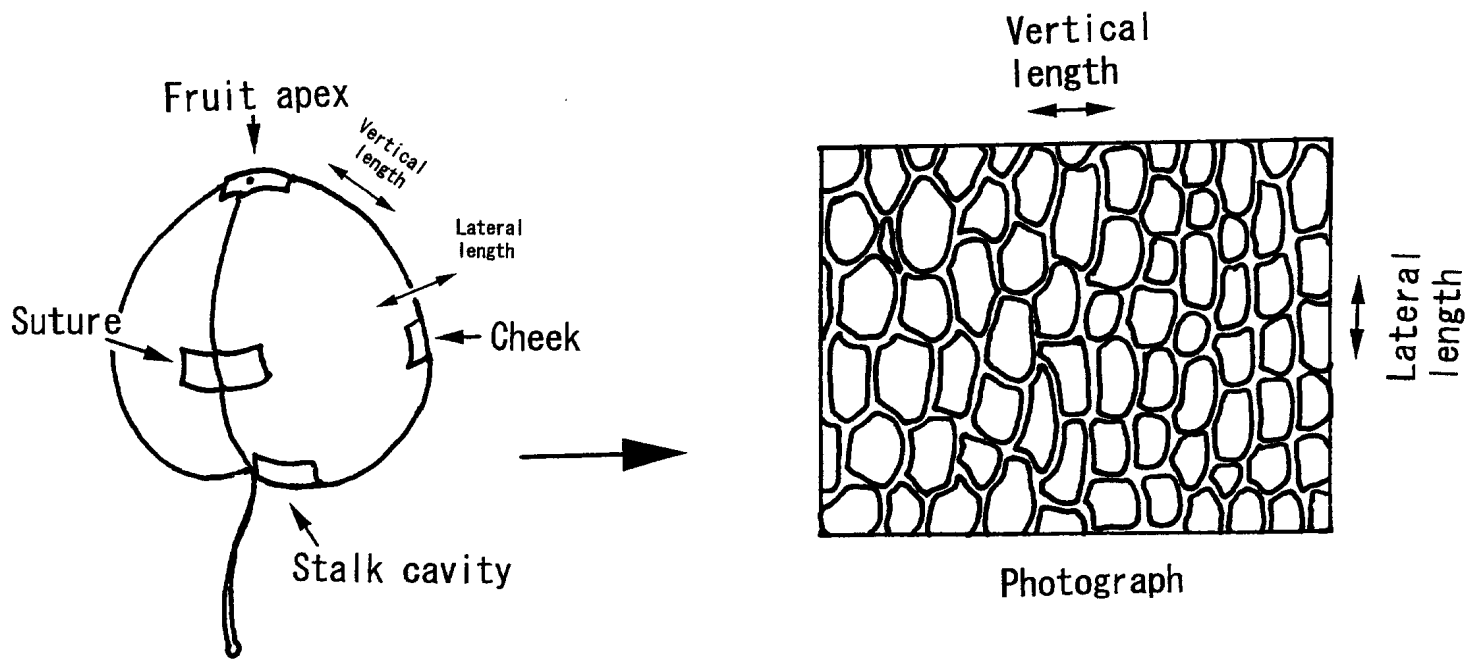

Fig. 2. Measurement sites for skin cell sizes. Skin at fruit apex, cheek, stalk cavity and suture was cut and made into hand sections, took photographs under microscope, and measured cell length in two directions. 
cell size was examined by measuring the vertical and lateral lengths of skin cells in the fruit apex, cheek, stalk cavity and suture as shown in Fig. 2. Each part was scraped with a razor, and photographed under an optical microscope and the length measuring the lengths of 30 representative cells parallel (vertical) and perpendicular to the suture (lateral) were measured. Cells on two mature, average-sized fruit were measured twice for each part. Force-deformation curves were obtained by using a rheometer (Fudoh, NRM-2010J), and the reading at bioyield point was determined as flesh firmness. Skin perpendicular to the cheek suture on 10 fruit was removed and the force at bioyield using a $\phi 3 \mathrm{~mm}-$ needle head with the speed of $60 \mathrm{~mm} / \mathrm{min}$ was calculated as a measure of flesh firmness. For the water immersion experiment in 1991, up to 45 mature fruit per cultivars were used to examine the increase in fruit weight, degree of cracking and rate of cracked fruit after a 12-, 24- and 48-hr immersion. Using these data, correlations among different parameters and annual differences in the values among them are established.

All the fruit were sampled from trees that were cultivated in the open field. To prevent cracking from rainfall, in 1990 and 1991, a part of the trees from which the fruit was harvested was covered with poly-vinylchloride sheets.

\section{Results}

Results of the cracking experiment using water immersion in 1990 and 1991 (Tables 1,2) reveal that in all

Table 1. Water absorption rate, degree of cracking and rate of cracked fruit by $12-, 24-$ and $48-\mathrm{hr}$ water immersion in sweet and sour cherry cultivars in 1990.

\begin{tabular}{|c|c|c|c|c|c|c|c|c|c|c|c|}
\hline \multirow[b]{2}{*}{$\begin{array}{l}\text { Cultivar or } \\
\text { selection }\end{array}$} & \multirow[b]{2}{*}{$\begin{array}{c}\text { Sampling } \\
\text { date }\end{array}$} & \multirow[b]{2}{*}{$\begin{array}{c}\text { Fruit } \\
\text { weight } \\
(\mathrm{g})\end{array}$} & \multicolumn{3}{|c|}{$12 \mathrm{hr}$} & \multicolumn{3}{|c|}{$24 \mathrm{hr}$} & \multicolumn{3}{|c|}{$48 \mathrm{hr}$} \\
\hline & & & $\begin{array}{c}\text { Water } \\
\text { absorption } \\
(\%)\end{array}$ & $\begin{array}{l}\text { Degree of } \\
\text { cracking }^{z}\end{array}$ & $\begin{array}{c}\text { Rate of } \\
\text { cracking }^{\mathrm{y}} \\
(\%)\end{array}$ & $\begin{array}{c}\text { Water } \\
\text { absorption } \\
(\%)\end{array}$ & $\begin{array}{l}\text { Degree of } \\
\text { cracking }\end{array}$ & $\begin{array}{c}\text { Rate of } \\
\text { cracking } \\
(\%)\end{array}$ & $\begin{array}{c}\text { Water } \\
\text { absorption } \\
(\%)\end{array}$ & $\begin{array}{l}\text { Degree of } \\
\text { cracking }\end{array}$ & $\begin{array}{c}\text { Rate of } \\
\text { cracking } \\
(\%)\end{array}$ \\
\hline P. cerasus $\times \operatorname{Pin}^{x}$ & $6 / 25$ & 2.9 & 3.0 & $0 \pm 0^{w}$ & 0 & 6.3 & $0.7 \pm 1.7$ & 20.0 & 13.0 & $4.3 \pm 2.7$ & 75.0 \\
\hline Valera & $6 / 19$ & 4.9 & 3.5 & $0 \pm 0.2$ & 5.0 & 6.5 & $0.7 \pm 1.5$ & 20.0 & 16.0 & $2.4 \pm 2.5$ & 60.0 \\
\hline Ebony & $6 / 17$ & 6.7 & 3.0 & $0 \pm 0.3$ & 10.0 & 5.5 & $0.4 \pm 0.7$ & 25.0 & 12.0 & $1.1 \pm 1.7$ & 45.0 \\
\hline Jumbonishiki & $6 / 14$ & 6.1 & 3.5 & $0.1 \pm 0.4$ & 5.0 & 5.0 & $0.2 \pm 0.5$ & 15.0 & 11.5 & $1.6 \pm 1.4$ & 15.0 \\
\hline Obako & $6 / 25$ & 5.5 & 3.0 & $0.1 \pm 0.5$ & 10.0 & 6.7 & $1.0 \pm 1.5$ & 30.0 & 17.0 & $3.4 \pm 2.0$ & 80.0 \\
\hline Venus & $6 / 19$ & 6.6 & 3.0 & $0.1 \pm 0.7$ & 5.0 & 6.5 & $0.9 \pm 1.6$ & 25.0 & 16.0 & $3.7 \pm 2.8$ & 65.0 \\
\hline Schwartze Venus & $6 / 14$ & 5.9 & 4.0 & $0.2 \pm 0.5$ & 30.0 & 8.5 & $0.6 \pm 0.9$ & 35.0 & 18.0 & $2.0 \pm 2.4$ & 45.0 \\
\hline Okitama- 6 & $6 / 14$ & 4.5 & 2.4 & $0.4 \pm 2.8$ & 30.0 & 5.0 & $0.8 \pm 1.4$ & 45.0 & 10.0 & $1.5 \pm 1.4$ & 75.0 \\
\hline $183^{v}$ & $6 / 25$ & 6.6 & 5.5 & $1.0 \pm 1.1$ & 55.0 & 10.0 & $2.3 \pm 1.4$ & 90.0 & 15.0 & $4.1 \pm 1.2$ & 100.0 \\
\hline Burbank & $6 / 14$ & 4.7 & 4.5 & $1.0 \pm 1.3$ & 55.0 & 9.5 & $1.7 \pm 1.5$ & 70.0 & 23.5 & $3.6 \pm 1.5$ & 95.0 \\
\hline $419^{v}$ & $6 / 14$ & 5.6 & 5.5 & $1.2 \pm 1.3$ & 80.0 & 10.0 & $2.3 \pm 1.6$ & 85.0 & 19.3 & $4.3 \pm 1.9$ & 90.0 \\
\hline $147^{\mathrm{v}}$ & $6 / 25$ & 6.4 & 5.5 & $1.2 \pm 1.3$ & 55.0 & 12.0 & $2.8 \pm 1.5$ & 95.0 & 24.5 & $5.0 \pm 1.3$ & 100.0 \\
\hline Kristin & $6 / 19$ & 6.3 & 3.5 & $1.3 \pm 1.8$ & 55.0 & 8.5 & $2.6 \pm 2.3$ & 70.0 & 20.5 & $5.1 \pm 2.5$ & 85.0 \\
\hline Chapman & $6 / 14$ & 4.3 & 5.5 & $1.6 \pm 2.0$ & 55.0 & 11.0 & $3.0 \pm 2.6$ & 65.0 & 23.5 & $4.2 \pm 2.1$ & 90.0 \\
\hline Rainier & $6 / 30$ & 7.6 & 5.0 & $1.9 \pm 1.3$ & 95.0 & 21.5 & $4.0 \pm 0.9$ & 95.0 & 24.0 & $5.6 \pm 1.2$ & 100.0 \\
\hline Gil Peck & $6 / 25$ & 6.7 & 5.0 & $2.5 \pm 2.0$ & 60.0 & 10.5 & $5.0 \pm 1.2$ & 75.0 & 17.5 & $6.6 \pm 0.7$ & 90.0 \\
\hline Ulster & $6 / 25$ & 8.2 & 5.0 & $3.0 \pm 1.4$ & 95.0 & 10.5 & $5.2 \pm 0.7$ & 100.0 & 20.5 & $6.5 \pm 0.5$ & 100.0 \\
\hline Early Richmond & $6 / 14$ & 4.7 & 11.0 & $3.2 \pm 2.3$ & 80.0 & 19.7 & $5.9 \pm 1.4$ & 95.0 & 32.5 & $6.9 \pm 0.4$ & 100.0 \\
\hline Napoleon & $6 / 25$ & 8.1 & 4.5 & $3.3 \pm 1.7$ & 100.0 & 10.0 & $4.6 \pm 1.4$ & 100.0 & 20.0 & $6.3 \pm 0.6$ & 100.0 \\
\hline Yeperia & $6 / 30$ & 6.5 & 6.0 & $3.5 \pm 1.1$ & 100.0 & 12.0 & $5.1 \pm 0.8$ & 100.0 & 22.5 & $6.1 \pm 0.4$ & 100.0 \\
\hline Hedelfinger & $6 / 14$ & 7.7 & 9.0 & $3.7 \pm 1.4$ & 100.0 & 14.0 & $4.4 \pm 1.2$ & 100.0 & 23.5 & $4.8 \pm 1.0$ & 100.0 \\
\hline Satonishiki & $6 / 19$ & 8.9 & 10.0 & $3.9 \pm 1.1$ & 100.0 & 16.0 & $6.2 \pm 0.7$ & 100.0 & 23.0 & $6.6 \pm 0.5$ & 100.0 \\
\hline Bing & $6 / 30$ & 6.8 & 8.5 & $4.0 \pm 0.7$ & 100.0 & 14.0 & $5.4 \pm 0.7$ & 100.0 & 18.5 & $6.1 \pm 0.6$ & 100.0 \\
\hline Van & $6 / 25$ & 9.5 & 8.5 & $4.3 \pm 1.1$ & 100.0 & 13.5 & $5.2 \pm 0.7$ & 100.0 & 16.1 & $6.1 \pm 0.4$ & 100.0 \\
\hline Vic & $6 / 25$ & 9.5 & 6.0 & $4.3 \pm 0.8$ & 100.0 & 12.5 & $5.4 \pm 0.7$ & 100.0 & 16.3 & $6.0 \pm 0.4$ & 100.0 \\
\hline $463^{v}$ & $6 / 25$ & 6.8 & 13.0 & $4.5 \pm 1.2$ & 100.0 & 25.0 & $6.2 \pm 0.6$ & 100.0 & 30.7 & $6.5 \pm 0.5$ & 100.0 \\
\hline $253^{v}$ & $6 / 14$ & 7.5 & 6.0 & $5.3 \pm 1.0$ & 100.0 & 9.5 & $6.0 \pm 0.5$ & 100.0 & 10.0 & $6.6 \pm 0.5$ & 100.0 \\
\hline $236^{\mathrm{v}}$ & $6 / 14$ & 8.2 & 8.5 & $5.3 \pm 1.4$ & 100.0 & 13.0 & $6.3 \pm 0.8$ & 100.0 & 14.5 & $6.7 \pm 0.5$ & 100.0 \\
\hline
\end{tabular}

${ }^{2}$ Degree of cracking $=($ scale point of 0 to $7 \times$ fruit number at each scale $) /$ total fruit number shown in Fig. 1 , and shown as rounding data.

${ }^{y}$ Percentage of fruit cracked over 1 shown in Fig. 1.

' 'Pin' is a cultivar belonging to Prunus pennsylvanica.

${ }^{\mathrm{w}}$ Standard deviation.

v Selections. Parentages are as follows, '183': open pollination of 'Satonishiki', '419': 'Bing' $\times$ 'Governer Wood', '147': open pollination of 'Napoleon', '463': unknown, '253': open pollination of 'Merton Glory', '236': open pollination of 'Rockport Bigarreau'. 
cultivars subjected to water immersion, water seeped into the fruit flesh with time and the degree of cracking and rate of cracked fruit increased. However, there were wide differences among cultivars in the water absorption rate, degree of cracking, and rate of cracked fruit. The 1990 experiment produced wide differences among cultivars in the increase of water absorption after $12 \mathrm{hr}$, from the lowest of $2.4 \%$ in 'Okitama- 6 ' to the highest of $13 \%$ in ' 463 .' These differences further widened with time. There was a linear relationship between the duration of water immersion and water absorption rate, but in cultivars with high water absorption rate, this

Table 2. Water absorption rate, degree of cracking and rate of cracked fruit by $12-, 24-$ and $48-\mathrm{hr}$ water immersion in sweet and sour cherry cultivars in 1991

\begin{tabular}{|c|c|c|c|c|c|c|c|c|c|c|c|c|}
\hline \multirow[b]{2}{*}{ Cultivar or selection } & \multirow[b]{2}{*}{$\begin{array}{c}\text { Sampling } \\
\text { date }\end{array}$} & \multirow[b]{2}{*}{$\begin{array}{l}\text { Fruit } \\
\text { weight } \\
\text { (g) }\end{array}$} & \multirow[b]{2}{*}{$\begin{array}{l}\text { Number } \\
\text { of fruit }\end{array}$} & \multicolumn{3}{|c|}{$12 \mathrm{hr}$} & \multicolumn{3}{|c|}{$24 \mathrm{hr}$} & \multicolumn{3}{|c|}{$48 \mathrm{hr}$} \\
\hline & & & & $\begin{array}{c}\text { Water } \\
\text { absorption } \\
(\%)\end{array}$ & $\begin{array}{l}\text { Degree of } \\
\text { cracking }^{2}\end{array}$ & $\begin{array}{c}\text { Rate of } \\
\text { cracking }^{y} \\
(\%)\end{array}$ & $\begin{array}{c}\text { Water } \\
\text { absorption } \\
(\%)\end{array}$ & $\begin{array}{l}\text { Degree of } \\
\text { cracking }\end{array}$ & $\begin{array}{c}\text { Rate of } \\
\text { cracking } \\
(\%)\end{array}$ & $\begin{array}{c}\text { Water } \\
\text { absorption } \\
(\%)\end{array}$ & $\begin{array}{l}\text { Degree of } \\
\text { cracking }\end{array}$ & $\begin{array}{c}\text { Rate of } \\
\text { cracking } \\
(\%)\end{array}$ \\
\hline Turkey Black Heart & $6 / 7$ & 3.7 & 45 & 3.5 & $0.1 \pm 0.3^{x}$ & 11.1 & 7.0 & $0.4 \pm 0.8$ & 35.6 & 11.0 & $0.9 \pm 1.2$ & 51.1 \\
\hline Jumbonishiki & $6 / 7$ & 6.2 & 21 & 3.0 & $0.1 \pm 0.5$ & 13.0 & 5.8 & $0.6 \pm 1.2$ & 34.4 & 12.9 & $1.9 \pm 2.2$ & 81.3 \\
\hline Valera & $6 / 20$ & 5.4 & 45 & 1.5 & $0.1 \pm 0.6$ & 6.7 & 3.5 & $0.8 \pm 1.4$ & 22.2 & 9.0 & $1.8 \pm 2.5$ & 44.4 \\
\hline Merton Heart & $6 / 7$ & 5.4 & 46 & 2.5 & $0.2 \pm 0.5$ & 13.3 & 5.0 & $0.4 \pm 1.0$ & 22.2 & 11.0 & $1.6 \pm 2.0$ & 44.4 \\
\hline Okitama- 6 & $6 / 11$ & 5.3 & 45 & 2.5 & $0.4 \pm 0.7$ & 26.7 & 4.5 & $1.2 \pm 1.2$ & 68.9 & 9.5 & $2.0 \pm 1.6$ & 84.4 \\
\hline P. cerasus $\times$ Pin $^{\mathrm{w}}$ & $6 / 27$ & 3.4 & 45 & 6.0 & $0.5 \pm 1.3$ & 15.6 & 12.0 & $2.2 \pm 2.6$ & 46.7 & 18.0 & $5.2 \pm 2.6$ & 60.0 \\
\hline Ebony & $6 / 17$ & 6.7 & 45 & 2.0 & $0.6 \pm 0.6$ & 51.1 & 5.0 & $0.7 \pm 0.9$ & 51.1 & 10.0 & $1.1 \pm 1.5$ & 55.6 \\
\hline $147^{\mathrm{v}}$ & $6 / 7$ & 8.9 & 45 & 4.0 & $0.6 \pm 0.8$ & 44.4 & 9.5 & $1.8 \pm 1.4$ & 82.2 & 21.0 & $4.9 \pm 1.5$ & 55.6 \\
\hline Venus & $6 / 20$ & 7.2 & 45 & 2.0 & $0.8 \pm 1.2$ & 42.2 & 6.5 & $2.7 \pm 2.4$ & 68.9 & 14.0 & $4.5 \pm 2.3$ & 88.9 \\
\hline Early Purple & $6 / 7$ & 2.8 & 45 & 5.0 & $0.8 \pm 1.2$ & 46.7 & 9.5 & $1.7 \pm 1.9$ & 57.8 & 15.5 & $3.0 \pm 1.3$ & 68.9 \\
\hline Compact Stella & $6 / 17$ & 6.4 & 45 & 2.5 & $1.1 \pm 0.7$ & 82.2 & 6.0 & $1.6 \pm 1.0$ & 93.3 & 12.0 & $3.0 \pm 1.5$ & 95.6 \\
\hline Schwartze Venus & $6 / 17$ & 8.7 & 45 & 2.5 & $1.1 \pm 1.2$ & 60.0 & 5.5 & $1.8 \pm 1.5$ & 82.2 & 14.0 & $3.4 \pm 2.0$ & 93.3 \\
\hline Obako & $6 / 20$ & 6.3 & 20 & 2.5 & $1.3 \pm 1.6$ & 45.0 & 7.5 & $3.3 \pm 2.0$ & 85.0 & 15.5 & $4.9 \pm 1.7$ & 95.0 \\
\hline Mazzard - 2 & $6 / 26$ & 3.4 & 44 & 4.5 & $1.6 \pm 2.2$ & 48.9 & 8.0 & $4.3 \pm 2.3$ & 91.1 & 12.0 & $5.6 \pm 2.0$ & 73.3 \\
\hline Early Richmond & $6 / 17$ & 5.2 & 45 & 6.0 & $1.7 \pm 2.5$ & 35.6 & 13.5 & $5.0 \pm 2.1$ & 86.7 & 22.5 & $6.1 \pm 2.0$ & 91.1 \\
\hline Kristin & $6 / 17$ & 7.6 & 45 & 3.0 & $1.9 \pm 1.3$ & 80.0 & 7.0 & $4.2 \pm 1.4$ & 100.0 & 17.5 & $5.9 \pm 0.8$ & 100.0 \\
\hline Manmat & $6 / 17$ & 8.1 & 45 & 3.0 & $2.0 \pm 0.9$ & 95.6 & 6.0 & $3.1 \pm 1.1$ & 97.8 & 14.5 & $4.2 \pm 1.1$ & 100.0 \\
\hline $183 \mathrm{v}$ & $6 / 27$ & 7.8 & 45 & 8.0 & $2.1 \pm 1.0$ & 93.3 & 11.5 & $3.1 \pm 0.7$ & 100.0 & 14.5 & $3.9 \pm 0.7$ & 100.0 \\
\hline Rainier & $6 / 27$ & 9.5 & 45 & 5.0 & $2.1 \pm 1.1$ & 100.0 & 9.5 & $4.0 \pm 1.0$ & 100.0 & 16.5 & $5.0 \pm 1.2$ & 100.0 \\
\hline Burbank & $6 / 17$ & 6.3 & 30 & 4.5 & $2.1 \pm 1.3$ & 83.3 & 9.0 & $4.1 \pm 0.8$ & 100.0 & 15.0 & $5.6 \pm 0.9$ & 100.0 \\
\hline Ulster & $6 / 27$ & 7.5 & 45 & 4.5 & $2.2 \pm 1.1$ & 88.9 & 8.5 & $3.4 \pm 1.1$ & 95.6 & 16.0 & $4.7 \pm 0.9$ & 100.0 \\
\hline Delikata & $6 / 7$ & 6.6 & 45 & 4.0 & $2.2 \pm 1.2$ & 100.0 & 6.0 & $3.8 \pm 1.2$ & 100.0 & 11.0 & $5.2 \pm 1.1$ & 100.0 \\
\hline Governer Wood & $6 / 11$ & 6.7 & 45 & 3.5 & $2.3 \pm 1.4$ & 66.7 & 7.5 & $5.1 \pm 1.3$ & 97.8 & 11.0 & $5.9 \pm 0.9$ & 100.0 \\
\hline $419^{\mathrm{v}}$ & $6 / 7$ & 6.8 & 30 & 7.0 & $2.6 \pm 1.0$ & 100.0 & 13.0 & $4.4 \pm 0.9$ & 100.0 & 22.0 & $6.1 \pm 0.8$ & 100.0 \\
\hline $455^{\mathrm{v}}$ & $6 / 7$ & 6.2 & 45 & 5.0 & $2.7 \pm 1.3$ & 97.8 & 11.5 & $5.8 \pm 1.2$ & 100.0 & 26.0 & $6.8 \pm 0.8$ & 100.0 \\
\hline Satonishiki & $6 / 17$ & 8.3 & 45 & 5.5 & $2.9 \pm 1.1$ & 100.0 & 11.5 & $4.7 \pm 1.0$ & 100.0 & 17.5 & $6.1 \pm 0.8$ & 100.0 \\
\hline Hedelfinger & $6 / 7$ & 7.8 & 45 & 6.5 & $3.1 \pm 0.7$ & 100.0 & 12.0 & $4.9 \pm 0.7$ & 100.0 & 19.0 & $5.9 \pm 0.2$ & 100.0 \\
\hline Vic & $6 / 17$ & 8.9 & 30 & 6.0 & $3.1 \pm 0.8$ & 100.0 & 11.0 & $4.4 \pm 0.7$ & 100.0 & 16.0 & $5.7 \pm 0.6$ & 100.0 \\
\hline Bing & $6 / 20$ & 7.2 & 45 & 4.5 & $3.1 \pm 0.9$ & 97.8 & 11.5 & $4.7 \pm 0.8$ & 100.0 & 15.0 & $5.7 \pm 0.6$ & 100.0 \\
\hline Napoleon & $6 / 27$ & 7.8 & 45 & 3.5 & $3.1 \pm 1.2$ & 100.0 & 8.0 & $4.6 \pm 0.9$ & 100.0 & 15.0 & $6.0 \pm 0.5$ & 100.0 \\
\hline Van & $6 / 20$ & 9.7 & 45 & 4.0 & $3.2 \pm 0.8$ & 100.0 & 7.5 & $4.2 \pm 0.7$ & 100.0 & 11.5 & $4.9 \pm 0.6$ & 100.0 \\
\hline Rockport Bigarreau & $6 / 11$ & 6.8 & 45 & 6.0 & $3.2 \pm 0.8$ & 100.0 & 12.5 & $5.1 \pm 0.8$ & 100.0 & 25.0 & $6.0 \pm 0.7$ & 100.0 \\
\hline Yeperia $^{u}$ & $6 / 27$ & 7.2 & 45 & 5.5 & $3.6 \pm 0.9$ & 97.8 & 12.0 & $4.5 \pm 0.6$ & 100.0 & 18.0 & $6.0 \pm 0.5$ & 100.0 \\
\hline Compact Lambert & $6 / 20$ & 9.2 & 30 & 5.5 & $3.6 \pm 1.1$ & 100.0 & 10.0 & $5.0 \pm 0.8$ & 100.0 & 15.0 & $5.6 \pm 0.8$ & 100.0 \\
\hline $214^{\mathrm{v}}$ & $6 / 27$ & 9.2 & 45 & 5.5 & $3.7 \pm 1.4$ & 93.3 & 11.0 & $5.5 \pm 0.8$ & 100.0 & 14.5 & $6.3 \pm 0.6$ & 100.0 \\
\hline $463^{\mathrm{v}}$ & $6 / 27$ & 8.4 & 30 & 15.0 & $4.4 \pm 1.1$ & 100.0 & 24.5 & $5.2 \pm 0.9$ & 100.0 & 24.5 & $5.6 \pm 0.8$ & 100.0 \\
\hline Gil Peck & $6 / 20$ & 8.8 & 45 & 4.5 & $4.4 \pm 1.2$ & 100.0 & 9.0 & $5.8 \pm 0.4$ & 100.0 & 16.0 & $6.7 \pm 0.5$ & 100.0 \\
\hline $236^{\mathrm{v}}$ & $6 / 11$ & 9.4 & 20 & 6.5 & $4.7 \pm 1.2$ & 100.0 & 11.5 & $6.1 \pm 0.5$ & 100.0 & 16.0 & $6.8 \pm 0.4$ & 100.0 \\
\hline $253^{\mathrm{v}}$ & $6 / 11$ & 7.5 & 45 & 8.5 & $4.8 \pm 1.1$ & 100.0 & 16.5 & $6.6 \pm 0.5$ & 100.0 & 23.5 & $7.0 \pm 0$ & 100.0 \\
\hline
\end{tabular}

${ }^{2}$ Degree of cracking $=($ scale point of 0 to $7 \times$ fruit number at each scale $) /$ total fruit number shown in Fig. 1.

${ }^{\mathrm{y}}$ Percentage of fruit cracked over 1 shown in Fig. 1.

${ }^{\mathrm{x}}$ Standard deviation.

w 'Pin' is a cultivar belonging to Prunus pennsylvanica.

v Selections. Parentages are as follows, '147': open pollination of 'Napoleon', '183': open pollination of 'Satonishiki', '419': 'Bing' $\times$ 'Governer Wood', '455': unknown, '214': open pollination of 'Satonishiki', '463': unknown, '236': open pollination of 'Rockport Bigarreau', 253': open pollination of 'Merton Glory'.

${ }^{\mathrm{u}}$ Data of skin cell sizes are not available. 
relationship was not maintained after $48 \mathrm{hr}$.

Likewise, there were clear differences in the degree of cracking among cultivars. ' $P$. cerasus $\times$ Pin,' 'Valera,' 'Ebony,' 'Jumbonishiki,' 'Obako,' 'Venus,' 'Schwartze Venus' and 'Okitama-6' expressed a degree of cracking of less than one, whereas, four cultivars had values of more than four. The ranking in the degree of cracking among cultivars and selections was maintained after 24 and $48 \mathrm{hr}$. However, some cultivars rapidly cracked and, in certain cases, the ranking of degree of cracking was reversed.

Differences in the rate of cracked fruit among cultivars were also confirmed in 1991, i.e., cultivars with a low degree of cracking remained low.

Differences in the water absorption rate, degree of cracking and rate of cracked fruit among the cultivars as well as changes with the duration of water immersion were similarly observed in 1990. 'Early Richmond', a sour cherry cultivar which is generally considered as having a low incidence of cracking, had a degree of cracking of 1.7 after $12 \mathrm{hr}$; but since its degree of cracking was 3.0 in 1990. This cultivar cannot be classified as crack-resistant but rated as an average crack-susceptible cultivar. An entirely crack-resistant cultivar was not found in this 2-year experiments.
The correlation coefficients among water absorption rate, degree of cracking and rate of cracked fruit were shown in Table3. $12 \mathrm{hr}$ after water immersion test in 1991 the $r$-value between water absorption rate and degree of cracking was 0.631 , whereas that between water absorption rate and rate of cracked fruit was 0.451 . Likewise, a significant, high positive correlation coefficient of 0.854 was obtained between degree of cracking and rate of cracked fruit. Similar trends were found in measurements taken after the $24-$ and $48-\mathrm{hr}$ immersion tests.

The yearly correlation calculated among the 28 cultivars used in 1990 and 1991, between the degree of cracking and rate of cracked fruit, $12 \mathrm{hr}$ after immersion, yielded $r$ values of 0.880 and 0.885 , respectively; the correlation for water absorption rate after $12 \mathrm{hr}$ between years was 0.706 , which is significant but comparatively lower than the other two parameters (Fig. 3). Similar trends were found in measurements taken after 24 and $48 \mathrm{hr}$ of water immersion. The foregoing results indicate for that water immersion, for evaluating cultivars crackresistance is relatively stable and dependable.

The measurement of the vertical and lateral lengths of the skin cells at fruit apex, cheek, stalk cavity and suture as well as the measurement result of the flesh firmness

Table 3. Correlation coefficients between fruit weight, water absorption rate, degree of cracking and rate of cracked fruit $12-$ and $24-\mathrm{hr}$ water immersion.

\begin{tabular}{lcccccc}
\hline & WA $^{2} 12$ & $\mathrm{DC}^{\mathrm{y}} 12$ & $\mathrm{CR}^{\mathrm{x}} 12$ & $\mathrm{WA} 24$ & $\mathrm{DC} 24$ & $\mathrm{CR} 24$ \\
\hline Fruit weight & $0.226 \mathrm{NS}$ & $0.664^{* *}$ & $0.718^{* *}$ & $0.206 \mathrm{NS}$ & $0.506^{* *}$ & $0.611^{* *}$ \\
WA12 & & $0.631^{* *}$ & $0.451^{* *}$ & $0.957^{* *}$ & $0.554^{* *}$ & $0.419^{* *}$ \\
DC12 & & & $0.854^{* *}$ & $0.648^{* *}$ & $0.914^{* *}$ & $0.776^{* *}$ \\
CR12 & & & & $0.444^{* *}$ & $0.788^{* *}$ & $0.905^{* *}$ \\
WA24 & & & & & $0.626^{* *}$ & $0.447^{* *}$ \\
DC24 & & & & & & $0.826^{* *}$ \\
\hline
\end{tabular}

${ }^{z}$ WA: water absorption rate.

${ }^{y}$ DC: degree of cracking.

${ }^{x} \mathrm{CR}$ : rate of cracked fruit.

NS, ${ }^{* *}$ Non - significant or significant at $P<0.01$, respectively.

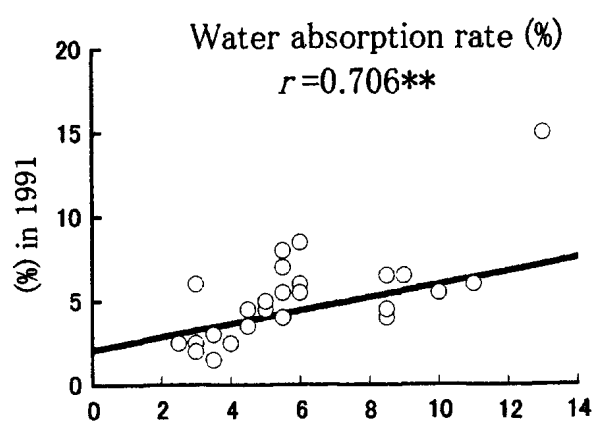

(\%) in 1990
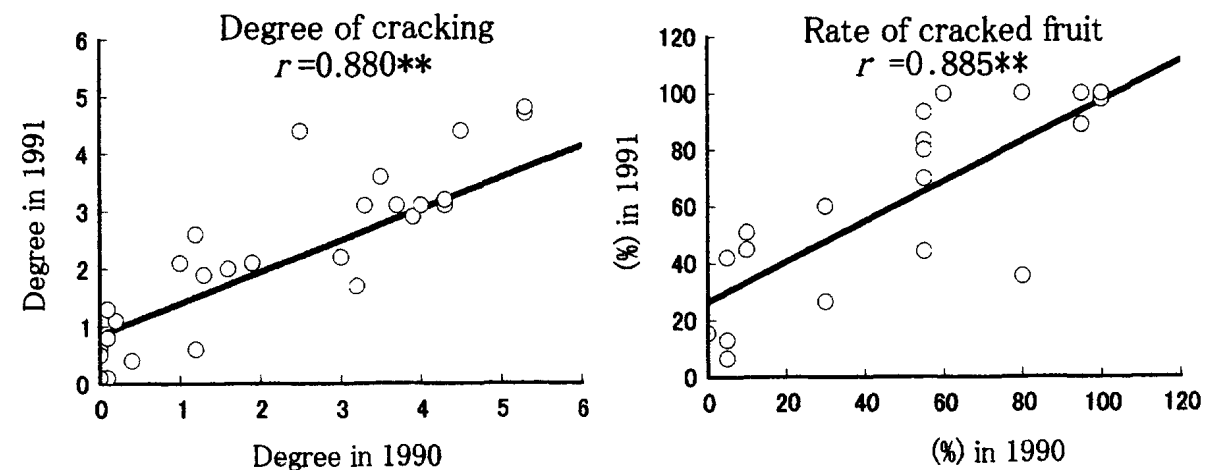

Fig. 3. Distribution plots showing yearly correlation between water absorption rate, degree of cracking and rate of cracked fruit $12-$ hr after water immersion for 28 cherry cultivars and selections. ${ }^{* *}$ Siginificant at $P<$ 0.01 . 
(Table 4) reveal that the average of vertical length of skin cell was $53.9 \mu \mathrm{m}$ at fruit apex, $44.1 \mu \mathrm{m}$ at cheek, $46.6 \mu \mathrm{m}$ at stalk cavity, and $55.4 \mu \mathrm{m}$ at suture. Those of the lateral lengths at each site were 53.3, 63.2, 40.9 and $42.8 \mu \mathrm{m}$, respectively. The largest standard deviation was observed in the vertical length at fruit apex and stalk cavity.

The sour cherry 'Early Richmond,' whose flesh firm- ness was measured at $29 \mathrm{~g}$, was the softest cultivar. A huge variance in flesh firmness occurred among sweet cherries ranging from $50 \mathrm{~g}$ in 'Turkey Black Heart,' 'Merton Heart,' 'Okitama-6,' to that exceeding $140 \mathrm{~g}$ 'Gil Peck,' '214' and '463.'

The $r$ values between the degree of cracking after 12 $\mathrm{hr}$ and fruit weight, flesh firmness, vertical and lateral lengths of the fruit apex, and lateral length of the cheek,

Table 4. Flesh firmness, lateral and vertical length of skin cell measured at four parts of sweet and sour cherries.

\begin{tabular}{|c|c|c|c|c|c|c|c|c|c|c|}
\hline \multirow[b]{2}{*}{ cultivar or selection } & \multirow{2}{*}{$\begin{array}{l}\text { Sampling } \\
\text { date }\end{array}$} & \multirow{2}{*}{$\begin{array}{l}\text { Flesh } \\
\text { firmness } \\
\text { (g) }\end{array}$} & \multicolumn{2}{|c|}{ Fruit apex } & \multicolumn{2}{|c|}{ Cheek } & \multicolumn{2}{|c|}{ Stalk cavity } & \multicolumn{2}{|c|}{ Suture } \\
\hline & & & $\begin{array}{l}\text { vertical } \\
(\mu \mathrm{m})\end{array}$ & $\begin{array}{l}\text { lateral } \\
(\mu \mathrm{m})\end{array}$ & $\begin{array}{l}\text { vertical } \\
(\mu \mathrm{m})\end{array}$ & $\begin{array}{l}\text { lateral } \\
(\mu \mathrm{m})\end{array}$ & $\begin{array}{l}\text { vertical } \\
(\mu \mathrm{m})\end{array}$ & $\begin{array}{l}\text { lateral } \\
(\mu \mathrm{m})\end{array}$ & $\begin{array}{l}\text { vertical } \\
(\mu \mathrm{m})\end{array}$ & $\begin{array}{l}\text { lateral } \\
(\mu \mathrm{m})\end{array}$ \\
\hline Merton Heart & $6 / 7$ & 52.2 & 31.09 & 38.90 & 37.80 & 62.03 & 50.94 & 31.74 & 59.70 & 41.45 \\
\hline Turkey Black Heart & $6 / 7$ & 51.8 & 36.34 & 41.38 & 43.79 & 62.40 & 37.07 & 47.07 & 48.85 & 36.27 \\
\hline Venus & $6 / 20$ & 56.5 & 38.24 & 39.26 & 37.22 & 52.03 & 38.17 & 37.07 & 52.62 & 36.78 \\
\hline Schwartze Venus & $6 / 17$ & 105.0 & 41.16 & 43.50 & 44.23 & 57.51 & 45.47 & 37.37 & 51.67 & 41.38 \\
\hline$P$. cerasus $\times \operatorname{Pin}^{2}$ & $6 / 27$ & 42.5 & 41.82 & 48.90 & 43.13 & 70.13 & 40.50 & 45.98 & 59.99 & 39.85 \\
\hline Early Purple & $6 / 7$ & 61.1 & 43.04 & 45.69 & 34.23 & 53.13 & 41.16 & 43.20 & 55.61 & 37.51 \\
\hline Compact Stella & $6 / 17$ & 62.0 & 45.90 & 42.84 & 32.40 & 51.67 & 38.75 & 34.15 & 53.28 & 38.10 \\
\hline Mazzard - 2 & $6 / 17$ & 34.5 & 46.34 & 51.38 & 35.10 & 62.76 & 39.94 & 37.44 & 48.82 & 36.20 \\
\hline Gil Peck & $6 / 20$ & 148.1 & 46.49 & 55.03 & 40.94 & 61.74 & 32.11 & 34.15 & 59.19 & 38.39 \\
\hline Okitama- 6 & $6 / 11$ & 53.4 & 47.95 & 48.09 & 36.49 & 56.56 & 39.63 & 29.92 & 57.65 & 45.69 \\
\hline Jumbonishiki & $6 / 7$ & 61.9 & 48.09 & 51.23 & 40.72 & 60.43 & 39.19 & 35.61 & 50.79 & 39.92 \\
\hline Manmat & $6 / 17$ & 78.9 & 50.58 & 55.17 & 37.95 & 59.62 & 42.77 & 40.29 & 53.35 & 35.98 \\
\hline $253^{y}$ & $6 / 11$ & 74.7 & 52.69 & 50.65 & 37.07 & 88.82 & 46.20 & 33.94 & 51.09 & 38.17 \\
\hline Early Richmond & $6 / 17$ & 29.1 & 53.13 & 43.42 & 50.72 & 57.73 & 53.71 & 38.31 & 56.63 & 53.49 \\
\hline $455^{\mathrm{y}}$ & $6 / 7$ & 78.8 & 54.95 & 56.04 & 43.35 & 68.30 & 44.26 & 46.93 & 55.54 & 42.18 \\
\hline $147^{\mathrm{y}}$ & $6 / 7$ & 66.2 & 55.61 & 61.16 & 48.31 & 62.40 & 46.63 & 50.65 & 51.01 & 47.36 \\
\hline Burbank & $6 / 17$ & 138.7 & 56.63 & 63.93 & 44.81 & 65.90 & 51.30 & 42.84 & 60.21 & 43.20 \\
\hline Satonishiki & $6 / 17$ & 87.0 & 57.07 & 55.68 & 43.42 & 62.18 & 53.20 & 44.52 & 57.58 & 45.54 \\
\hline Hedelfinger & $6 / 7$ & 78.3 & 57.87 & 55.25 & 47.95 & 68.02 & 51.30 & 42.33 & 59.84 & 42.22 \\
\hline Ulster & $6 / 27$ & 87.5 & 58.17 & 57.80 & 48.75 & 60.57 & 46.56 & 45.47 & 46.63 & 42.11 \\
\hline Bing & $6 / 20$ & 58.7 & 58.38 & 54.22 & 37.51 & 57.58 & 46.27 & 36.71 & 62.76 & 50.06 \\
\hline $214^{\mathrm{y}}$ & $6 / 27$ & 143.0 & 59.33 & 53.05 & 43.35 & 66.41 & 43.79 & 47.15 & 60.28 & 41.96 \\
\hline Governer Wood & $6 / 11$ & 67.5 & 60.65 & 56.34 & 55.46 & 64.44 & 59.33 & 40.50 & 52.76 & 51.38 \\
\hline Rockport Bigarreau & $6 / 11$ & 73.1 & 60.94 & 59.04 & 51.38 & 59.55 & 58.53 & 41.89 & 51.89 & 45.39 \\
\hline $236^{\mathrm{y}}$ & $6 / 11$ & 60.9 & 61.74 & 53.64 & 44.52 & 65.68 & 36.85 & 33.06 & 50.36 & 36.85 \\
\hline $183^{y}$ & $6 / 25$ & 89.8 & 61.89 & 65.61 & 52.69 & 73.20 & 71.37 & 46.85 & 67.36 & 45.76 \\
\hline Obako & $6 / 20$ & 62.2 & 61.89 & 57.87 & 52.47 & 70.72 & 27.88 & 42.69 & 58.01 & 43.93 \\
\hline Van & $6 / 20$ & 122.4 & 63.93 & 65.39 & 52.25 & 69.84 & 58.09 & 44.44 & 58.68 & 45.02 \\
\hline $463^{y}$ & $6 / 27$ & 140.4 & 64.44 & 53.20 & 44.74 & 66.27 & 42.18 & 41.45 & 59.99 & 44.15 \\
\hline Delikata & $6 / 7$ & 59.9 & 65.17 & 58.97 & 53.35 & 66.78 & 66.63 & 51.01 & 58.60 & 44.30 \\
\hline Cmpact Lambert & $6 / 20$ & 83.3 & 68.09 & 62.91 & 48.53 & 64.59 & 55.68 & 48.09 & 52.69 & 41.16 \\
\hline Rainier & $6 / 27$ & 81.3 & 68.89 & 56.78 & 44.08 & 66.34 & 39.26 & 37.37 & 40.43 & 54.01 \\
\hline $419^{y}$ & $6 / 7$ & 70.0 & 69.11 & 58.82 & 55.54 & 79.90 & 65.97 & 37.00 & 60.14 & 54.15 \\
\hline Vic & $6 / 17$ & 105.1 & 63.05 & 59.04 & 49.92 & 67.43 & 53.79 & 37.07 & 57.73 & 45.61 \\
\hline Average & & 85.5 & 53.94 & 53.25 & 44.14 & 63.17 & 46.59 & 40.94 & 55.41 & 42.83 \\
\hline SD & & 24.8 & 9.72 & 7.08 & 6.17 & 5.68 & 9.71 & 5.30 & 5.13 & 5.16 \\
\hline
\end{tabular}

2 'Pin' is a cultivar belonging to Prunus pennsylvanica.

${ }^{y}$ Selections. Parentages are as follows, '147': open pollination of 'Napoleon', '183': open pollination of 'Satonishiki', '419': 'Bing $\times$ Governer Wood', ‘455': unknown, ‘214': open pollination of 'Satonishiki', ‘463': unknown, '236': open pollination of 'Rockport Bigarreau', '253': open pollination of 'Merton Glory'. 
Table 5. Correlation coefficients among water absorption rate, degree of cracking, rate of cracked fruit $12 \mathrm{hr}$ after water immersion, flesh firmness and cell length at four parts of the fruit.

\begin{tabular}{lcccccccccc}
\hline \hline & $\begin{array}{c}\text { Fruit } \\
\text { weight }\end{array}$ & $\begin{array}{c}\text { Flesh } \\
\text { firmness }\end{array}$ & $\begin{array}{c}\mathrm{VL}^{\mathrm{w}} \text { at } \\
\text { apex }\end{array}$ & $\begin{array}{c}\mathrm{LL}^{\mathrm{v}} \text { at } \\
\text { apex }\end{array}$ & $\begin{array}{c}\mathrm{VL} \text { at } \\
\text { Cheek }\end{array}$ & $\begin{array}{c}\text { LL at } \\
\text { Cheek }\end{array}$ & $\begin{array}{c}\mathrm{VL} \text { at } \\
\text { Cavity }\end{array}$ & $\begin{array}{c}\text { LL at } \\
\text { Cavity }\end{array}$ & $\begin{array}{c}\mathrm{VL} \text { at } \\
\text { Suture }\end{array}$ & $\begin{array}{c}\text { LL at } \\
\text { Suture }\end{array}$ \\
\hline Fruit weight & - & $0.567^{* *}$ & $0.595^{* *}$ & $0.490^{* *}$ & $0.311 \mathrm{NS}$ & $0.257 \mathrm{NS}$ & $0.226 \mathrm{NS}$ & $-0.001 \mathrm{NS}$ & $0.032 \mathrm{NS}$ & $0.234 \mathrm{NS}$ \\
Flesh firmness & $0.567^{* *}$ & - & $0.310 \mathrm{NS}$ & $0.324^{*}$ & $0.160 \mathrm{NS}$ & $0.283 \mathrm{NS}$ & $0.292 \mathrm{NS}$ & $0.128 \mathrm{NS}$ & $0.310 \mathrm{NS}$ & $0.107 \mathrm{NS}$ \\
$\mathrm{WA}^{z} 12$ & $0.226 \mathrm{NS}$ & $0.384^{*}$ & $0.474^{* *}$ & $0.305 \mathrm{NS}$ & $0.261 \mathrm{NS}$ & $0.462^{* *}$ & $0.265 \mathrm{NS}$ & $0.089 \mathrm{NS}$ & $0.222 \mathrm{NS}$ & $0.261 \mathrm{NS}$ \\
$\mathrm{DC}^{y} 12$ & $0.664^{* *}$ & $0.515^{* *}$ & $0.649^{* *}$ & $0.515^{* *}$ & $0.255 \mathrm{NS}$ & $0.393^{*}$ & $0.228 \mathrm{NS}$ & $-0.066 \mathrm{NS}$ & $0.160 \mathrm{NS}$ & $0.173 \mathrm{NS}$ \\
$\mathrm{DR}^{\mathrm{x}} 12$ & $0.718^{* *}$ & $0.503^{* *}$ & $0.763^{* *}$ & $0.669^{* *}$ & $0.337^{*}$ & $0.362^{*}$ & $0.428^{* *}$ & $0.149 \mathrm{NS}$ & $0.151 \mathrm{NS}$ & $0.321^{*}$ \\
\hline
\end{tabular}

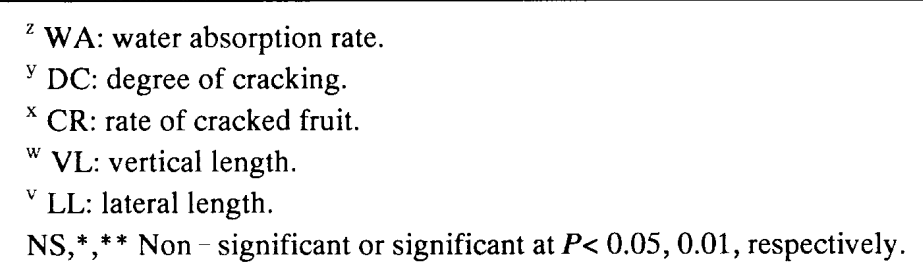

were: $0.664,0.515,0.649,0.515$ and 0.393 , respectively (Table 5). The $r$ values between rate of cracked fruit after a 12-hr immersion and fruit weight and flesh firmness were 0.718 and 0.503 , respectively. Except for the lateral length of the stalk cavity and the suture, the rate of cracked fruit correlated significantly with six parameters of the skin cells. Our data also show that high $r$ values $0.763,0.669$, respectively, exist between cracked fruit and the vertical and lateral lengths at the fruit apex. Likewise, $r$ values between the water absorption rate and the flesh firmness, fruit apex vertical length and cheek lateral length were $0.384,0.474$ and 0.462 , respectively, but that between the rate of water absorption and fruit weight was not as definitive.

The above results indicate that cracking of cherry is closely related to fruit weight, among other parameters, as well as flesh firmness, skin cell sizes, especially their vertical and lateral lengths at the fruit apex. The contribution rate calculated from multiple regression analysis of degree of cracking after $12 \mathrm{hr}$ to fruit weight, flesh firmness, numerical values of the skin cell lengths of eight cultivars is $69.2 \%$. When the numerical values of the skin cell lengths are deleted, the contribution rate from those of the fruit weight, flesh firmness and vertical cell length at the apex amounts to $57.3 \%$, while that of all other parameters to the rate of cracked fruit after $12 \mathrm{hr}$ is $78.8 \%$. The contribution rate of numerical values of fruit weight, flesh firmness and vertical length of cells at the apex is $70.7 \%$.

\section{Discussion}

We found that cracking susceptibility of cherry fruit is influenced by the skin cell sizes in this study. Sawada (1931) demonstrated that stomata are distributed more on the upper half of the fruit. Iizuka and Yano (1974) suggested that moisture is absorbed from the stomata that open up with ripening. In contrast, Glenn and Poovaiah (1989) who showed that water absorption into the fruit occurs through the cuticular layer, expressed doubts about the role of stomata in this process. Sekse (1995), and Børve and Sekse (2000) demonstrated that the cuticular fracture near the abscission scar of stigma accompanies fruit growth as we also observed. These data and our result suggest that water is absorbed mainly through the rupture. Yamamoto et al. (1990b) found out that stress increased during the the rapid growth period, with the longitudinal stress concentrated in the apex and stalk cavity and the latitudinal stress in the cheek. They also found a significant correlation at peak time between the degree of surface cracking versus the daily average rate of change of the fruit length and distortion from growth (Yamamoto et al., 1996).

The correlation between skin cell length at fruit apex and cracking was the highest (Table 5), while the lateral length of cheek cells was the largest (Table 4). Which indicates that the stress of fruit growth was concentrated only in the radial direction from a stigma scar at fruit apex. A large standard deviation among vertical cell length at fruit apex probably contributes to the varietal differences in cracking susceptibility.

The relationship between flesh firmness and cracking susceptibility is conflicting (Kertesz and Nebel, 1935; Yamamoto et al., 1996; Roser, 1996). In this experiment, a significant positive correlation was established between the two parameters (Table 5), confirming the trends observed by Kertesz and Nebel (1935). Flesh firmness of cherry fruit is affected by the degree of methyl esterification of the pectin-side chain and thickness of cell wall and lamella (Batisse et al., 1996). Water absorption by the mesocarp cells is related more to the colloidal properties of cell walls than to the osmotic pressure of the cell plasma (Christensen, 1972b). The information suggesting the relationship between water absorption and flesh pectin in cherry supports our findings. However, the contribution rate of flesh firmness to the rate of cracked fruit is only $25 \%$ so that this relationship may not be based on strong evidence but on the cultivar used. 
The $r$ value of 0.632 between rate of water absorption and degree of cracking $12 \mathrm{hr}$ after water immersion (Table 3), accounts for a $40 \%$ of the former to the latter. Therefore, the degree of cracking susceptibility cannot be solely explained on the basis the quantity of water absorpted because fruit growth depends on water uptake and the plasticity of epidermal cells; the relatively smaller skin cells have greater extensibility potential that may effectively inhibit fruit cracking.

Fruit weight correlates highly with the degree of cracking and rate of cracked fruit (Table 5), as well as with the vertical and lateral lengths of skin cells at the apex, which indicates that fruit weight has an influence on cracking susceptibility.

In conclussion, our results indicate that cracking susceptibility has significant correlation with fruit weight, flesh firmness, and vertical and lateral lengths of skin cells at apex. When taken individually, these parameters do not adequately explain the variance of cracking susceptibility among cultivars, but when the multiple regression analysis is applied, extremely high contribution rates are obtained to suggest that cracking susceptibility involves multiple factors.

There are some cultivars that have low cracking susceptibility, especially with small fruit size and very soft flesh or those with relatively firm flesh but smaller skin cell sizes at the fruit apex. From the perspective of breeding, soft flesh and small, crack-resistant fruit are not desirable traits. However, the findings that small skin cells at the apex are resistant to splitting indicate one direction for breeding crack-resistant cultivars. Hence, by selecting those cultivars with small skin cell diameters, especially vertical length at the apex in ripe fruit, it may be possible to breed crack-resistant cultivars with large firm flesh.

\section{Literature Cited}

Andersen, P. C. and D. G. Richardson. 1982. A rapid method to estimate fruit water status with special reference to rain cracking of sweet cherries. J. Amer. Soc. Hort. Sci. 107: $441-444$.

Batisse, C., M. Buret and P. J. Coulomb. 1996. Biochemical differences in cell wall of cherry fruit between soft and crisp fruit. J. Agric. Food Chem. 44: 453-457.

Børve, J. and L. Sekse. 2000. Cuticlar fracture promote postharvest fruit rot in sweet cherries. Plant Disease 84 : 1180-1184.

Christensen, J. V. 1972a. Cracking in cherries. III . Determination of cracking susceptibility. Acta. Agric. Scandinavica 22: $128-136$.

Christensen, J. V. 1972b. Cracking in cherries. IV . Physiological studies of the mechanism of cracking. Acta. Agric. Scandinavica 22: 153-162.
Davenport, D. C., K. Uriu and R. M. Hagan. 1972. Antitranspirant film: Curtailing intake of external water by cherry fruit to reduce cracking. HortScience 7: 507508.

Glenn, G. M. and B. W. Poovaiah. 1989. Cuticular properties and post harvest calcium applications influence cracking of sweet cherries. J. Amer. Soc. Hort. Sci. 114: 781 -788 .

Iizuka, I. and K. Yano. 1974. Water absorption of detached sweet cherries. Bull. Yamagata Univ. Agric. Sci. 7: 209 -216 .

Kertesz, Z. I. and B. B. Nebel. 1935. Observations on the cracking of sweet cherries. Plant Physiol. 10: 763-772.

King, J. and R. A. Norton. 1987. Cracking resistance in certain cherry cultivars and selection. Fruit Varieties J. 41: 83-84.

Lane, W. D., M. Meherriuk and D. L. McKenzie. 2000. Fruit cracking of a susceptible, an intermediate, and a resistant sweet cherry cultivar. HortScience 35: 239-242.

Roser, I. 1996. investigation on cracking susceptibility of sweet cherry cultivars. Acta. Hortic. 410: 331-337.

Sawada, E. 1931. Distribution of stomata on cherry fruit surface. Agric. and Hortic. 10: 485-501. (In Japanese).

Sekse, L. 1995. Cuticular fracturing in fruit of sweet cherry (Prunus avium L.) resulting from changing soil water contents. J. Amer. Soc. Hort. Sci. 70: 631-635.

Tucker, L. R. 1934. A varietal study of the susceptibility of sweet cherries to cracking. Univ. Idaho Agric. Exp. Sta. Bull. 211: 3-19.

Verner, L. and E. C. Blodgett. 1931. Physiological studies of the cracking of sweet cherries. Univ. Idaho Agric. Exp. Sta. Bull. 184: 3-15.

Wade, N. L. 1988. Effects of metabolic inhibitors on cracking of sweet cherry fruit. Scientia Hortic. 34: 239 248.

Yamamoto, T., M. Kudo and S. Watanabe. 1990a. Fruit cracking and characteristics of fruit thickening in 'Satonishiki' cherry. J. Japan. Soc. Hort. Sci. 59: 325332. (In Japanese with English summary).

Yamamoto, T., K. Hosoi and S. Watanabe. 1990b. Relationship between the degree of fruit cracking of sweet cherries and the distribution of surface stress of the fruit analyzed by a newly developed system. J. Japan. Soc. Hort. Sci. 59: 509-517. (In Japanese with English summary).

Yamamoto, T., E. Sugai and T. Niida. 1996. Interrelationship between the characterisitcs of fruit growth and the cracking susceptibility in apple and sweet cherry cultivars. J. Japan. Soc. Hort. Sci. 64: 789-799. (In Japanese with English summary).

Zielinski, Q. B. 1964. Resistance of sweet cherry varieties to fruit cracking in relation to fruit and pit size and fruit color. Proc. Amer. Soc. Hort. Sci. 84: 98-102. 
オウトウ品種・系統の裂果感受性に及ぼす果実表皮細胞径および果肉硬度の影響

\author{
山口正己 ${ }^{*} \cdot$ 佐藤 功・石黒 亮 \\ 山形県立園芸試験場 991-0043 山形県寒河江市
}

\begin{abstract}
摘要
オウトウの裂果抵抗性品種・系統を明らかにするとともに, 裂果感受性に関係する要因を明らかにするために，甘果オウ トウ 37, 酸果オウトウおよび酸果オウトウとPrunus pensylvanica の雑種それぞれ 1, 計 39 品種·系統について水浸漬法 による裂果程度, 裂果率および水の吸収率と果実重, 果肉硬 度, 果頂部, 赤道部, 梗あ部および縫合線部における表皮細 胞の縦径および横径との関係について検討を行った. 裂果程 度および裂果率は水浸漬後時間の経過とともに上昇したが, 品種・系統による差異が認められた。すなわち, 'Turkey Black Heart', 'Jumbonishiki', 'Okitama-6', 'Ebony', 'Venus', 'Valera'などの品種・系統は裂果程度, 裂果率とも 小さな值を示したが, 'Napoleon', 'Van', 'Compact Lambert', 'Vic’などの品種では著しい裂果が認められた。果実 重, 果肉硬度, 果実の表皮細胞の縦径および横径にも大きな 品種間差異が認められた. 水浸漬 12 時間後の裂果程度と果 実重, 果肉硬度, 果頂部の表皮細胞繸径および横径との間に は, それぞれ $r=0.664,0.515,0.649,0.515$ 之有意な正の相

関が認められた。

また, 同じく 12 時間後の裂果率は果実重, 果肉硬度, 果頂 部の表皮細胞緃径および横径との間で $r=0.718,0.503$, 0.763，0.669 とそれぞれに有意な正の相関が認められた。こ のことから, 裂果感受性には果実重, 果肉硬度とともに表皮 細胞の大きさ, 特に果頂部の細胞の大きさが強く関係してい ると考元られた，吸水率は, 果肉硬度, 果頂部表皮細胞の繸 径との間で有意な正の相関が得られた。裂果率, 裂果程度と 測定項目間の重相関係数を算定したところ, 全測定項目との 間で 78.8，69.0\%の高い寄与率が得られた。 また, 果実重, 果肉硬度, 果頂部縦径亡の間でも 70.7, 57.3\%の高い寄与率 が得られたことから，これら 3 つの測定項目により裂果の感 受性を推定することが可能であると考えられた．また，28品 種系統について裂果程度, 裂果率, 吸水率の年次間の相関を みたところ，それぞれ $r=0.885 ， 0.880 ， 0.706$ と有意な高い相 関が得られたことから, 本試験で行った水浸漬法による裂果 程度の判定が有効であると考えられた。
\end{abstract}

